\title{
FUNÇÕES DE KOZAK ET AL. PARA AFILAMENTO DE CORDIA GOELDIANA HUBER EM PLANTIO HOMOGÊNEO NO MUNICÍPIO DE VILHENA - RO
}

\author{
Martha Aguiar Santos ${ }^{1}$ \\ Ronaldo Drescher ${ }^{2}$ \\ Rômulo Môra ${ }^{3}$ \\ Diogo Guido Streck Vendrusculo ${ }^{4}$
}

\section{INTRODUÇÃO}

O Setor Florestal Brasileiro, nos últimos anos tornou-se um dos mais relevantes no cenário mundial ao destacar tamanha relevância com quase oito milhões de hectares de árvores plantadas e responsável pela maioria de toda a madeira produzida para fins industriais no Brasil. O setor representou em 2015, por meio do Produto Interno Bruto (PIB), a arrecadação de R\$ 69,1 bilhões, representando aumento de 3,0\% em relação ao ano anterior e superior a outros setores, como o agrícola e o das indústrias, na economia brasileira (IBÁ, 2016).

A pressão sobre os recursos naturais tende a crescer exponencialmente ocasionado pelas necessidades básicas de consumo humano. Uma alternativa que auxilia para minimizar a exploração das matas nativas é o cultivo de plantios homogêneos usados para suprir e abastecer as indústrias de base florestal.

Contudo, algumas espécies utilizadas em plantios homogêneos ainda são pouco estudadas, como é o caso da Cordia goeldiana conhecida popularmente como freijó que apresenta uma multiplicidade de uso e excelente valor comercial, tem sido bastante

1 Eng. Florestal, Mestranda em Ciências Florestais e Ambientais da Universidade Federal de Mato Grosso, Cuiabá, Mato Grosso, Brasil. - E-mail: marthaguiar@gmail.com

2 Dr., Eng. Florestal, Departamento de Engenharia Florestal Universidade Federal de Mato Grosso, Cuiabá, Mato Grosso, Brasil. -E-mail: ronaldodrescher@gmail.com

3 Dr. Eng. Florestal. Departamento de Engenharia Florestal Universidade Federal de Mato Grosso, Cuiabá, Mato Grosso, Brasil. - E-mail: romulomef@yahoo.com.br

4 Engenheiro Florestal - PROTERRA - Projetos Agropecuários e Florestal. Porto Esperidião - MT. E-mail: diogoguido@hotmail.com 
utilizada nos programas de reflorestamento, no entanto muito pouco se sabe sobre o afilamento do fuste.

A diversidade florestal brasileira inclui muitas espécies de alto valor comercial, sendo assim imprescindível que hajam estudos relativos ao emprego de modelos estatísticos para quantificar multiprodutos da madeira (ANDRADE \& SCHMITT, 2017).

As equações de afilamento são uma das ferramentas que podem ser utilizadas para estimativas de produção, são flexíveis e estimam com precisão a produção por classes de sortimento para os mais variados fins. Assim, o conhecimento dessas informações auxilia na tomada de decisão sobre intervenções no plantio e no manejo florestal em geral.

Segundo Môra (2015), na quantificação da produção florestal, principalmente a de madeira serrada, deve-se considerar sempre a maximização da produção, diminuindo desperdício causado pela transformação da madeira, seja ela primária ou secundária. Nesse sentido, as funções de afilamento, que também são conhecidas como funções de taper, funções de adelgaçamento, modelos de perfil ou, ainda, funções de forma, são uma maneira de descrever matematicamente o perfil de um fuste (SCOLFORO, 2004).

A partir dessas funções, é possível estimar a altura onde um determinado diâmetro ocorre e o volume de partes do fuste, ou seja, quantificam os multiprodutos da madeira (madeira serrada, celulose, lenha entre outros), permitindo maior aproveitamento e agregação de valor ao produto. Além disso, as estimativas fornecidas por esses modelos fornecem informações que auxiliam na meIhor utilização do produto florestal, e no planejamento da colheita.

Árvores com mesmo diâmetro e mesma altura podem apresentar volumes e sortimentos distintos se a forma dos fustes for diferente (Scolforo et al., 1998). Isso impulsiona os estudos sobre a forma das árvores objetivando alcançar maior acurácia nas estimativas de sortimento ou de volume total ou parcial do fuste (KOZAK ET AL., 1969; AHRENS E HOLBERT, 1981; QUEIROZ ET AL., 2008; YOSHITANI JUNIOR, 2012; MÔRA, 2015).

Primeiramente surgiram os modelos de afilamento não segmentados, que utilizam uma única equação para descrever todo perfil do fuste. Depois, com a modernização e adven- 
to dos computadores e softwares estatísticos, surgiram os modelos segmentados que diferiam dos não segmentados por poderem dividir a árvore em três ou mais segmentos utilizando pontos de inflexão. E para melhorar o desempenho das funções de afilamento em relação ao ajuste e a utilização de pontos de inflexão, Kozak (1988) introduziu os modelos de afilamento de forma variável, que utilizam modelos de regressão não segmentados que de forma implícita dividem a árvore em três segmentos, sem necessidade do auxílio de pontos de inflexão. (MÔRA, 2015). Diante deste contexto, ao testar e selecionar funções de afilamento não segmentadas e de forma variável para um plantio homogêneo de Cordia goeldiana Huber. (Freijó) no município de Vilhena - RO e também, selecionar dentre as equações testadas qual explica melhor a forma do tronco e avaliar a eficiência da equação selecionada, para estimar o volume das árvores constitui-se de fundamental importância científica para a ciência em geral.

\section{DELINEAMENTO EXPERIMENTAL}

O estudo foi realizado na propriedade Schummann, localizada no município de Vilhena-RO, divisa com Mato Grosso, distante 700 km da capital, Porto Velho e 750 km de Cuiabá. O local da pesquisa apresenta as coordenadas geográficas $60^{\circ} 4^{\prime} 21.216^{\prime \prime}$ longitude Oeste e $12^{\circ} 50^{\prime} 2.55^{\prime \prime}$ latitude Sul de Greenwich (Figura 1).

A área pertence ao aquífero Parecis, bacia do rio Roosevelt contida na bacia Amazônica. A vegetação encontrada na área é de transição de Savana Estacional Arborizada sem floresta de galeria com Floresta Estacional semidecidual submontana com dossel emergente (RONDÔNIA, 2000). O solo predominante na região é do tipo Latossolo Vermelho-Amarelo, com areia distrófica (Rondônia, 2000), classificados como Latossolos Vermelhos Distróficos segundo o Sistema Brasileiro de Classificação de Solos (EMBRAPA, 2006).

Segundo Köppen, o clima da região é do tipo Aw (BASTOS E DINIZ, 1982). A precipitação total anual de Vilhena é de $2.068 \mathrm{~mm}$, sendo a estação chuvosa de outubro a abril, com média de precipitação mensal de $263 \mathrm{~mm}$. A temperatura média máxima anual é de $29^{\circ} \mathrm{C}$ e a mínima média é de $19,3^{\circ} \mathrm{C}$ e altitude de aproximadamente 612 m (RAMALHO et al., 2004). 


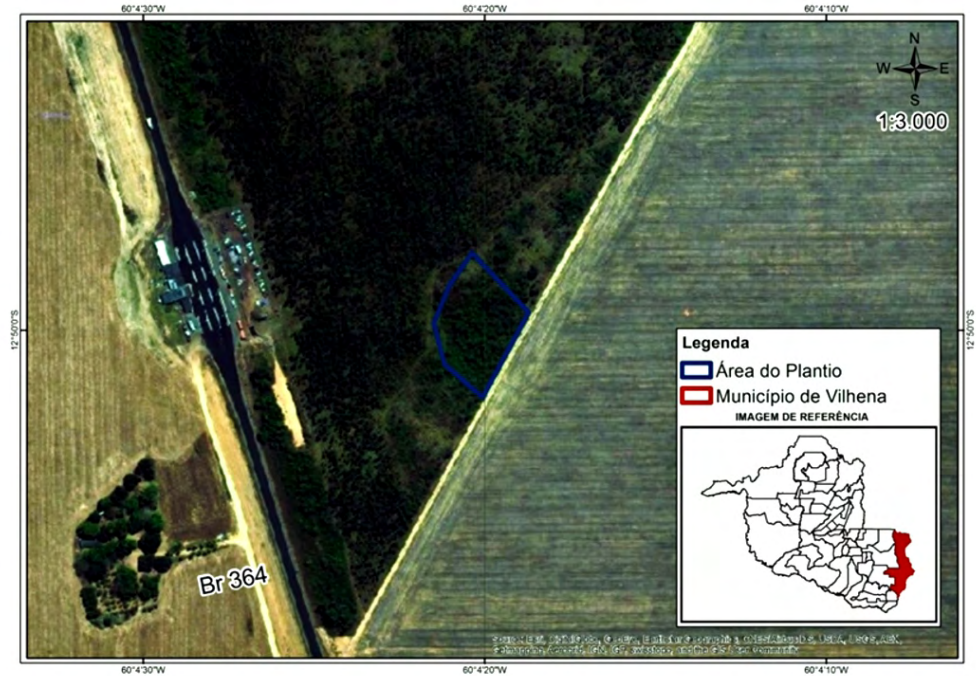

Figura 1. Mapa de localização da área de estudo

Fonte: Google Maps. 2017.

\section{Coleta de dados}

O estudo foi realizado em um plantio homogêneo e equiâneo de Cordia goeldiana de 17 anos e origem seminal, implantado em uma área de aproximadamente 0,6 ha com espaçamento $2 \times 2 \mathrm{~m}$. Não sofreu nenhuma intervenção silvicultural, como desrama ou podas de condução e desbastes. Também não foi efetuada correção de solo e fertilizações na área.

Foram selecionadas 15 árvores conforme o critério de dominância de Assman, para o método de cubagem rigoroso absoluto, através do método de Smalian para obtenção do volume. As árvores selecionadas foram abatidas. Sobre a árvore no chão foi esticada uma trena para medir a altura total e foram retirados discos de 3 a $5 \mathrm{~cm}$ de espessura em tais alturas: 0,$2 ; 0,5 ; 0,8 ; 1,3$; e a cada 1,0 $m$ até o diâmetro mínimo de 3,0 centímetros. Em cada disco será descrito a altura de coleta no tronco e o número da árvore com lápis carbono.

Os discos (Figura 2) foram transportados até o Laboratório de Crescimento e Produção na UFMT, onde foram mantidos para secagem por 15 dias em local à sombra e bem arejado. Após a secagem, 
foi realizado o lixamento da face de medição de cada disco, com máquina e lixa número 80 , a fim de facilitar a visualização dos anéis para aplicação da metodologia de análise de tronco tradicional.

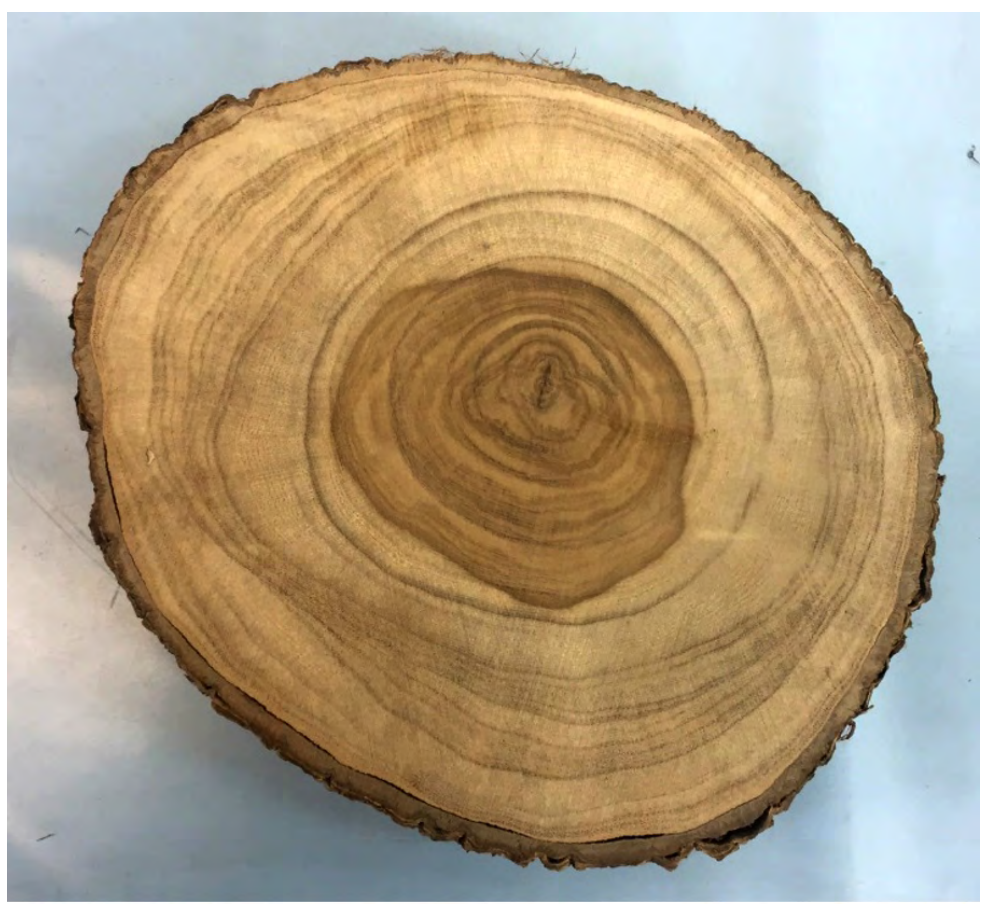

Figura 2. Disco de Cordia goeldiana H. lixado para análise de tronco

Os diâmetros de cada anel de crescimento foram medidos usando a metodologia de Barusso (1977), a qual vem sendo adotada em grande parte dos trabalhos de análise de tronco (Finger, 1992; Machado et al., no prelo), que se utilizam-se basicamente lápis e régua graduada. E para o cálculo do volume total em cada idade, foi utilizado o método de Smalian:

$$
V=\frac{g 1+g 2}{2} x L
$$

Em que: $V=$ volume total do tronco ou secção, em $\mathrm{m}^{3} ; \mathrm{g} 1=$ área transversal da parte inferior da seção; $\mathrm{g} 2$ = área transversal da parte superior da seção, ambos em $\mathrm{m}^{2} ; \mathrm{L}=$ comprimento das seções, em metros. 


\section{Ajuste das equações não segmentadas e de forma variável}

Para os ajustes foram utilizados o banco de dados das idades formado pelos dados de 6 a 12 anos. Foram testadas as funções de afilamento não segmentadas listadas na Tabela 1, propostas por KOZAK et al. (1969).

\begin{tabular}{ll}
\hline $\begin{array}{l}\text { Kozak et al } \\
(1969 \mathrm{a})\end{array}$ & $\left(\frac{\mathrm{d}_{i j}}{\mathrm{D} j}\right)^{2}=\beta_{1}\left(T_{i j}-1\right)+\beta_{2}\left(T_{i j}{ }^{2}-1\right)+\varepsilon_{i j}$ \\
\hline $\begin{array}{l}\text { Kozak et al } \\
(1969 \mathrm{~b})\end{array}$ & $\left(\frac{\mathrm{d}_{i j}}{\mathrm{D} j}\right)^{2}=\beta_{1}\left(1-2 T_{i j}+T_{i j}{ }^{2}\right)+\varepsilon_{i j}$ \\
\hline $\begin{array}{l}\text { Kozak et al } \\
(1969 \mathrm{c})\end{array}$ & $\left(\frac{d_{i j}}{D j}\right)^{2}=\beta_{0}+\beta_{1} T_{i j}+\beta_{2} T_{i j}{ }^{2}+\varepsilon_{i j}$ \\
\hline
\end{tabular}

Tabela 1 - Funções de afilamento não segmentadas testadas para Cordia goeldiana $\mathrm{H}$.

Onde: $d_{i j}=$ diâmetro à altura hi do tronco $D j=$ diâmetro à altura do peito; $T_{i j}=h_{j} /$ $H j ; h i=$ altura até uma seção "i" $n$ fuste, em m; $H j=$ altura total; $B i=$ parâmetros a serem estimados; $\varepsilon_{i j}=$ Erro aleatório

Foram testadas as funções afilamento de forma variável listadas na Tabela 2 também propostas por KOZAK et al (1969).

\begin{tabular}{|c|c|}
\hline$\frac{\text { Kozak }}{(1988)}$ & 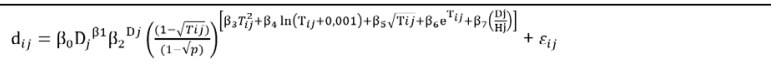 \\
\hline $\begin{array}{l}\text { Kozak (1994) } \\
\text { citado por Kozak } \\
\text { (1997) }\end{array}$ & $\mathrm{d}_{i j}=\beta_{0} \mathrm{D}_{j}{ }^{\beta 1} \beta_{2}{ }^{\mathrm{D} j}\left(\frac{(1-\sqrt{T i j})}{(1-\sqrt{ } p)}\right)^{\beta_{3}+\beta_{4} T_{i j}^{\frac{1}{4}}+\beta_{5} T_{i j}{ }^{\frac{1}{3}+\beta_{6} T_{i j} j^{1 / 2}+\beta_{7} \arcsin \left(1-T_{i j} j^{1 / 2}\right)+\beta_{8}}\left[\frac{1}{\frac{\mathrm{D} j}{\mathrm{H} j}+T_{i j}}\right]+\beta_{9} H_{j}}+\varepsilon_{i j}$ \\
\hline $\begin{array}{l}\text { Kozak } \\
\text { (1995) citado } \\
\text { Por Kozak (1997) }\end{array}$ & $\mathrm{d}_{i j}=\beta_{0} \mathrm{D} j^{\beta_{1}} \mathrm{H}^{\beta_{2}}\left(\frac{(1-\sqrt{T i j)}}{(1-\sqrt{p})}\right)\left[\beta_{3}\left(\frac{(1-\sqrt{T i j)}}{(1-\sqrt{p})}\right)^{\frac{1}{10}}+\beta_{4}\left(\frac{h_{i j}}{H_{i j}}\right)^{4}+\beta_{5} \arcsin \left(1-\sqrt{\frac{h_{i j}}{H_{i j}}}\right)+\beta_{6}\left(\frac{1}{D_{j}} e^{\frac{1}{H_{j}}}\right)+\beta_{7} \mathrm{D} j\left(\frac{(1-\sqrt{T(j)})}{(1-\sqrt{p})}\right)\right]+\varepsilon_{i j}$ \\
\hline Kozak (2004a) & $\mathrm{d}_{i j}=\beta_{0} D_{j}^{\beta_{1}}\left(\frac{1-T_{i j}{ }^{1 / 4}}{1-p^{1 / 4}}\right)^{\beta_{2}+\beta_{3}\left(1 / e^{D_{i} / H_{j}}\right)+\beta_{4} D_{j}}\left(\frac{1-T_{i j} j^{1 / 4}}{1-p^{1 / 4}}\right)+\beta_{3}\left(\frac{1-T_{i j}^{1 / 4}}{1-p^{1 / 4}}\right)^{\frac{D_{j}}{H_{j}}}+\varepsilon_{i j}$ \\
\hline$\frac{\text { Kozak }}{(2004 b)}$ & $\begin{aligned} \mathrm{d}_{i j}= & \beta_{0} D_{j}{ }^{\beta 1} H_{j}{ }^{\beta 2}\left(\frac{1-T_{i j}{ }^{1 / 3}}{1-p^{1 / 3}}\right)^{\left[\beta_{3} T_{i j}{ }^{4}+\beta_{4}\left(1 / e^{D_{j} / H j}\right)+\beta_{5}\left(\frac{1-T_{i j} j^{1 / 3}}{1-p^{1 / 3}}\right)^{0,1}+\beta_{6}\left(1 / D_{j}\right)+\beta_{7} H_{j}{ }^{1-T_{i j}}{ }^{1 / 3}+\beta_{8}\left(\frac{1-T_{i j}{ }^{1 / 3}}{1-p^{1 / 3}}\right)\right]} \\
& +\varepsilon_{i j}\end{aligned}$ \\
\hline
\end{tabular}

Tabela 2 - Funções de afilamento de forma variável testadas para Cordia goeldiana $\mathrm{H}$.

Onde: $d_{i j}=$ diâmetro à altura hi do tronco $\mathrm{Dj}=$ diâmetro à altura do peito; $T_{i j}=h_{j} /$ $H j$; $h i=$ altura até uma seção "i" n fuste, em m; $H j$ = altura total; $B i=$ parâmetros a serem estimados; $p=$ esse valor corresponde ao primeiro ponto de inflexão calculado no modelo de Max e Burkhart (1976), que corresponde quando a forma do fuste passa de neiloide para paraboloide e $\varepsilon_{i j}=$ Erro aleatório 
Para o cálculo do valor $p$ foi utilizada a função segmentada de Max e Burkhart (1976), utilizando o valor correspondente ao primeiro ponto de inflexão do modelo. O resultado obtido foi de 0,06530669 através do ajuste da função segmentada de MAX e BURKHART (1976).

\section{Processamentos dos dados}

No pré-processamento e consistência dos dados foi utilizado o Microsoft Excel por possuir ferramentas de fácil manuseio e para os ajustes dos modelos foi utilizado o software R (R Core Team, 2015). Através do Método iterativo através da técnica nls (Nonlinear Least) para obtenção dos coeficientes médios utilizando o algoritmo de Gauss-Newton implementado no software utilizado.

Foi utilizado o teste t para avaliar a significância dos coeficientes das equações, testando-se a hipótese nula do coeficiente ser igual a zero, utilizando um nível de significância de 5\%. Quando pelo menos um dos coeficientes dos modelos tiveram resultado não significativo, o modelo foi descartado do estudo. Os modelos ajustados foram utilizados para estimar o diâmetro e o volume total das árvores.

\section{Parâmetros para seleção dos modelos}

Para seleção do modelo mais acurado foram observados os seguintes critérios estatísticos:

- Coeficiente de determinação $\left(R^{2} \%\right)$;

- $\quad$ Erro padrão da estimativa em percentagem $\left(\mathrm{S}_{\mathrm{yx}} \%\right)$;

- Análise gráfica dos resíduos.

Segundo Machado et al. (2002), o erro padrão da estimativa $\left(\mathrm{S}_{\mathrm{yx}} \%\right)$ deve ser mais próximo de zero, o coeficiente de determinação $\left(\mathrm{R}^{2} \%\right)$ mais próximo de $100 \%$ e a análise gráfica dos resíduos. Para o cálculo desse critério utilizou-se a seguinte equação:

$$
\boldsymbol{R}^{2} \%=\left(\frac{\text { sQres }}{\text { sQtot }}\right) * 100
$$

Em que: $\mathrm{R}^{2}$ = coeficiente de determinação; SQres = soma de quadrados do resíduo; SQtot = soma de quadrados total. 
O erro padrão da estimativa em porcentagem $\left(\mathrm{S}_{\mathrm{yx}} \%\right)$ informa o erro médio ocasionado pelo uso do modelo (Soares et al., 2011), havendo a necessidade de recalcular o erro para as equações não lineares. Utilizou-se a seguinte equação:

$$
\boldsymbol{S}_{\boldsymbol{y} x} \%=\sqrt{\frac{Q M r e s}{\bar{\gamma}}} * 100
$$

Onde: $\mathrm{S}_{\mathrm{yx}} \%$ = erro padrão da estimativa em porcentagem; QMres = quadrado médio do resíduo; = média dos diâmetros observadas.

A análise gráfica dos resíduos permite detectar se há ou não tendenciosidade na estimativa da variável dependente ao longo da linha de regressão ou se os resíduos são independentes. Para a construção dos gráficos que foram utilizados na análise, calculou-se os valores residuais através da expressão:

$$
\operatorname{Resíduo~}(\%)=\frac{(\mathrm{Y}-\hat{\mathrm{Y}})}{\mathrm{Y}} * 100
$$

Em que: $\hat{Y}=$ valores estimados pela equação; $\mathrm{e} Y=$ valores observados.

Para avaliar a eficiência das funções de afilamento, em estimar o volume das árvores, como método comparativo, foi ajustado o modelo de Schumacher e Hall não linear, muito utilizado para estimativa de volume:

$$
\boldsymbol{V}=\beta 0 * d a p^{\beta 1} h t^{\beta 2}+\varepsilon
$$

Em que: $d a p=$ diâmetro à altura do peito; $h t=$ altura total; $B i=$ parâmetros a serem estimados; $\varepsilon_{i j}=$ erro aleatório.

\section{RESULTADOS E DISCUSSÃO}

\section{Ajuste dos modelos não segmentados e de forma variável para o diâmetro}

A partir dos ajustes realizados, foram obtidos os parâmetros estimados para os oito modelos que são apresentados na Tabela 3. 
De forma geral os modelos testados apresentaram coeficientes de determinação $\left(R^{2}\right)$ superiores a $94 \%$ e baixos valores de erro padrão das estimativas $\left(\mathrm{S}_{\mathrm{yx}}\right)$, variando entre $11,20 \%$ e $15,05 \%$, indicando que as equações foram eficientes e explicaram apropriadamente as estimativas de diâmetro ao longo do fuste. Segundo Favalessa (2012) essas estatísticas refletem a qualidade dos modelos e demonstram a correlação entre as variáveis independentes e a variável dependente.

Observando-se a Tabela 3, nota-se que a maioria dos coeficientes foram significativos, porém o modelo de forma variável de Kozak (1995), os coeficientes $\beta_{2}, \beta_{5}$ e $\beta_{7}$ foram não significativos a $5 \%$ de probabilidade pelo teste t. O modelo Kozak (2004b), também de forma variável obteve $\beta_{4}$ não significativo, portanto, não foi utilizado no estudo.

Os valores de $\mathrm{R}^{2}$ foram mais altos para as equações de forma variável, se comparado com as funções não segmentadas. Isso foi verificado por Môra (2015), quando afirma que as equações de forma variável são mais acuradas que as equações dos grupos segmentados e não segmentados. Estas equações tem o menor grau de viés e maior acurácia nas previsões de afilamento (Newnham, 1988, 1992; Kozak, 1988; Perez et al., 1990) entre outros.

Observa-se que o modelo de Kozak (1994) apresentou o maior valor de $R^{2}(96,50 \%)$, indicando que este explica de forma mais acurada a variação dos dados quando comparado aos demais. Nota-se também que esse modelo possui menor $S_{x y}$ $(11,20 \%)$, por se tratar de uma função de forma variável e possuir maior número de coeficientes, além de aproximar-se mais a forma do fuste.

Andrade (2014) ao testar 18 modelos de afilamento para Eucalyptus grandis e Eucalyptus urophylla, sendo seis não segmentados, dois segmentados e dez de forma variável, concluiu que os de forma variável, resultaram em estimativas mais acuradas para a variável diâmetro.

O modelo com menor coeficiente de determinação $\left(R^{2}\right)$ foi o de Kozak (1969c), com 94,43\% e consequentemente o maior erro $(15,01 \%)$. Esses valores são justificáveis, por se tratar de uma função não segmentada, com poucos coeficientes, o que lhe confere menor flexibilidade em relação as demais. 
Apesar das equações apresentarem um alto coeficiente de determinação $\left(R^{2}\right)$ as funções de Kozak (1969a) e Kozak (1969c), obtiveram erros de $14,56 \%$ e $15,051 \%$, respectivamente, o que pode estar relacionado a variação de idade (de 6 a 12 anos) das árvores do conjunto de dados provenientes da análise de tronco apresentarem. As demais equações obtiveram erros entre $11,20 \%$ e $13,74 \%$.

\begin{tabular}{|c|c|c|c|c|c|c|c|c|c|c|c|c|}
\hline \multirow{2}{*}{ Equação } & \multicolumn{10}{|c|}{ Coeficientes estimados } & \multirow{2}{*}{$\begin{array}{c}R^{2} \text { zjus } \\
(\%)\end{array}$} & \multirow{2}{*}{$\begin{array}{l}S_{y x} \\
(\%)\end{array}$} \\
\hline & $\beta_{0}$ & $\beta_{1}$ & $\beta_{2}$ & $\beta_{3}$ & $\beta_{4}$ & $\beta_{5}$ & $\beta_{6}$ & $\beta_{7}$ & $\beta_{\mathrm{g}}$ & $\beta_{9}$ & & \\
\hline Kozak (1969a) & & $-3,10258^{*}$ & $1,55352^{*}$ & & & & & & & & 95,26 & 14,56 \\
\hline Kozak (1969b) & & $1,42868^{*}$ & & & & & & & & & 95,26 & 13,74 \\
\hline Kozak (1969c) & & $1,55857^{*}$ & $-3,20628^{*}$ & $1,69468^{*}$ & & & & & & & 94,43 & 15,05 \\
\hline Kozak (1988) & $2,05495^{*}$ & $0,59714^{*}$ & $1,02646^{*}$ & $2,90048^{*}$ & $-0,85897^{*}$ & $6,24281^{*}$ & $-2,98324^{*}$ & $-0,30214^{*}$ & & & 96,35 & 11,41 \\
\hline Kozak (1994) & $2,18611^{*}$ & $0,57154^{*}$ & $1,02629^{\circ}$ & $-150,274^{*}$ & $334,654^{*}$ & $-316,246^{*}$ & $132,056^{*}$ & $89,1517^{*}$ & $1,30989^{*}$ & $-0,01311^{*}$ & 96,50 & 11,20 \\
\hline Kozak (1995) & $1,54354^{*}$ & $0,84643^{*}$ & $0,00866^{\mathrm{ns}}$ & $0,23330^{*}$ & $0,45563^{*}$ & $-0,03537^{\mathrm{ns}}$ & $0,89079^{*}$ & $-0,00497$ ns & & & 95,90 & 12,12 \\
\hline Kozak (2004a) & $1,55535^{*}$ & $0,84801^{*}$ & $0,12451^{*}$ & $1,37999^{*}$ & $0,02349^{*}$ & $-0,68151^{*}$ & & & & & 95,81 & 12,20 \\
\hline Kozak (2004b) & $1,42526^{*}$ & $0,99036^{*}$ & $-0,17694^{*}$ & $-0,20477^{*}$ & $-0,23287$ ns & $0,74735^{*}$ & $4,27671^{*}$ & $0,15640^{*}$ & & & 96,34 & 11,48 \\
\hline
\end{tabular}

Tabela 3 - Coeficientes obtidos nos ajustes das equações não-segmentadas e de forma variável para Cordia goeldiana $\mathrm{H}$. e estatísticas para avaliar as estimativas dos diâmetros

* significativo a $5 \%$ de probabilidade pelo teste $t$.

${ }^{\text {ns }}$ não significativo a $5 \%$ de probabilidade pelo teste

Outra análise utilizada para seleção do modelo mais acurado é o gráfico dos resíduos. Por meio desta percebe-se que a maioria dos modelos apresentam tendenciosidade ao longo da linha média, tendo uma dispersão de resíduos semelhantes (Figura 3).

Apenas o modelo de Kozak (1969c), apresentou uma tendência de superestimar os menores diâmetros. Os resíduos dos diâmetros estimados pelas demais equações, demonstram que a medida que os diâmetros se aproximam do ápice, mais subestimadas foram as estimativas.

A maioria das funções de afilamento presentes na literatura, tem essa dificuldade de estimar os diâmetros conforme próximos da ponta do fuste. Figueiredo et al. (2006) também verificaram a dificuldade na estimativa dos diâmetros nas maiores alturas, em todos os modelos que testou.

Mesmo que a equação de Kozak (1994) tenha apresentado as melhores estatísticas de ajuste ( $R^{2}$ e Syx) em relação as demais, e menor dispersão residual, observou-se que esta apresentou uma certa dificuldade em estimar os menores diâmetros. 

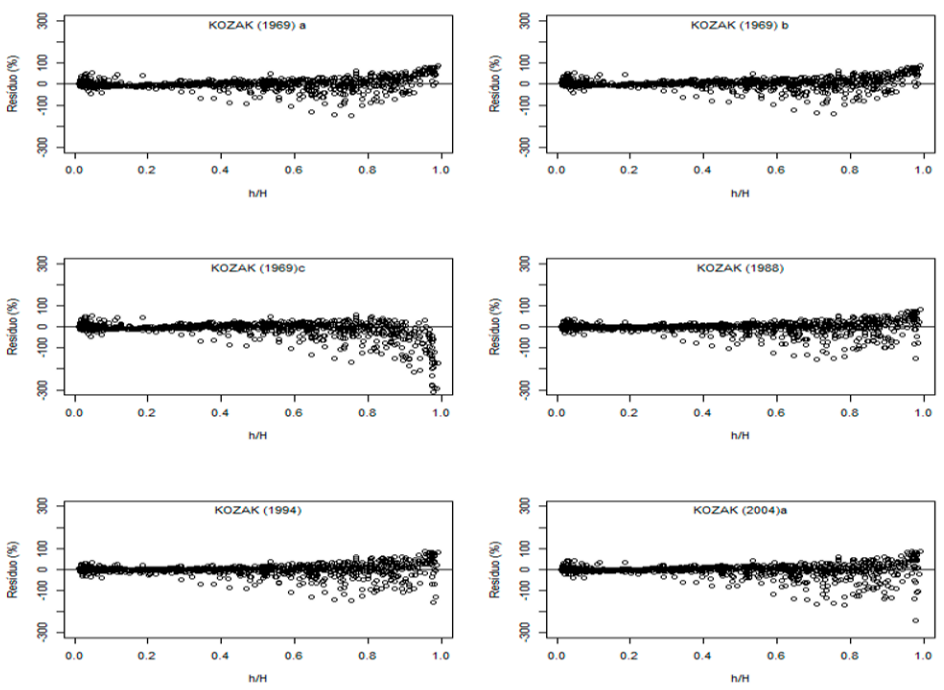

Figura 3. Distribuição dos resíduos (\%) em função de $h / h$ das estimativas de diâmetro das funções de afilamento não segmentadas e de forma variável para o povoamento de Cordia goeldiana $\mathrm{H}$, Vilhena, RO

Para comparação entre os diâmetros ao longo do fuste e os diâmetros estimados pelas equações Kozak (1994) e Kozak et al. (1969c) selecionadas como mais e a menos acurada, respectivamente, foi elaborado os perfis de árvore média real e estimada (Figura 4). E a diferença do volume estimado revela a importância de se testar funções que melhor se adequam aos dados. Pois a diferença na estimativa pode interferir diretamente no volume do produto final estimado.

Analisando o perfil da árvore média real e estimada pelo modelo de Kozak (1994) observa-se que as estimativas tenderam a subestimar os diâmetros em quase todas as alturas, com exceção da base e do ápice (Figura 4A). Na altura de $11 \mathrm{~m}$ percebe-se que as linhas se cruzam, mostrando que apesar do modelo ter apresentado tendências residuais nas maiores alturas, quando analisado para a árvore média, essa tendência foi minimizada. 0 modelo subestimou em 9,87\% os diâmetros da árvore média.

Enquanto que o modelo considerado como menos acurado de Kozak et al. (1969c), superestimou os diâmetros ao longo do fuste $(22,08 \%)$, principalmente na base (Figura 4B). Como descrito na 
literatura, os modelos não segmentados caracterizam-se pela simplicidade dos ajustes, mas em geral não explicam com propriedade as deformações que existem na base das árvores (ASSIS, 2001; QUEIROZ et al, 2008; FIGUEIREDO et al., 2006).
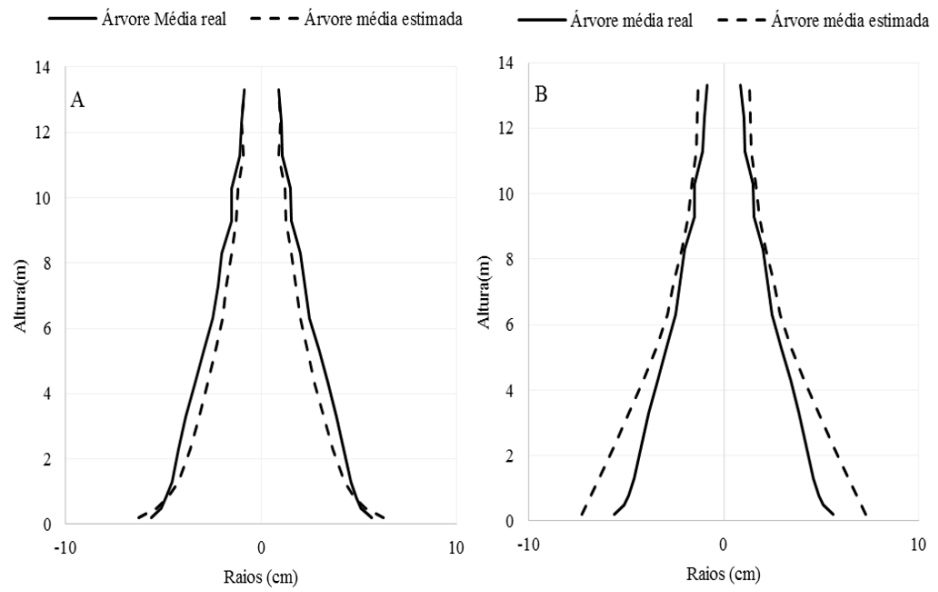

Figura 4. Árvores média real e estimada pela equação Kozak (1994) (A) e Kozak (1969c) (B) para o povoamento de Cordia goeldiana $\mathrm{H}$. Vilhena, RO

\section{Ajuste dos modelos não segmentados e de forma variável para volume}

Dentre os estudos existentes para afilamento, poucos abordam as estimativas volumétricas, quando, na realidade, um modelo que estime diâmetros satisfatoriamente não fornece necessariamente estimativas satisfatórias de volumes (Assis et al., 2005). Também segundo Campos e Leite (2009), o melhor modelo ajustado depende dos objetivos propostos para estudo, pois enquanto um modelo tem melhores resultados para estimar a variável diâmetro, ele pode não ser o melhor para estimar as variáveis altura e volume.

Sendo assim, foram testados para a estimativa de volume, apenas as funções que obtiveram coeficientes significativos, a partir do teste $t$, na estimativa de diâmetro, a fim de avaliar se as equações se ajustam de forma semelhante, ou não, na estimativa de diâmetro e volume. As estimativas do volume foram comparadas por meio da integração da expressão do diâmetro 
Os valores de erro padrão da estimativa foram acima de $10 \%$ em todas as funções, observando um menor valor na equação de forma variável Kozak (2004a), 11,39 \% (Tabela 4). Apesar de que o modelo de Kozak (1994) tenha sido o que melhor ajustou o diâmetro, o mesmo não apresentou o menor erro no ajuste de volume, sendo de 12,46 \%. Os erros de Kozak (1969a) e Kozak (1969c) foram praticamente os iguais (Tabela 4).

\begin{tabular}{ccccccc}
\hline EQUAÇÃO & $\begin{array}{c}\text { Kozak } \\
(1969 a)\end{array}$ & $\begin{array}{c}\text { Kozak } \\
(1969 b)\end{array}$ & $\begin{array}{c}\text { Kozak } \\
(1969 c)\end{array}$ & $\begin{array}{c}\text { Kozak } \\
(1988)\end{array}$ & $\begin{array}{c}\text { Kozak } \\
(1994)\end{array}$ & $\begin{array}{c}\text { Kozak } \\
(2004 a)\end{array}$ \\
\hline$S_{\mathrm{yx}}(\%)$ & 17,78 & 11,89 & 17,78 & 12,15 & 12,46 & 11,39 \\
\hline
\end{tabular}

Tabela 4-Erro padrão da estimativa de volume total $\left(\mathrm{S}_{\mathrm{yx}} \%\right)$ no ajuste das equações não-segmentadas e de forma variável para Cordia goeldiana $\mathrm{H}$.

Pela análise gráfica dos resíduos a função de Kozak (2004a) demonstrou uma adequada distribuição dos resíduos (Figura 5), apesar de ainda subestimar os menores volumes caracterizados pelos menores diâmetros.

Embora os valores de erro padrão da estimativa das funções não segmentadas e de forma variável são superiores aos valores obtidos pelos ajustes da equação de volume de Schumacher e Hall (10,65\%), a estimativa de volume através de funções de afilamento apresenta vantagens, pois Téo et al. (2012) citando Scolforo (1993) afirma que é possível quantificar o volume total da árvore, e quaisquer outros volumes comercias, que se estendem ao longo do fuste, de maneira distinta, além do volume total.

Sendo assim, a função de afilamento de forma variável proposta por Kozak (1994) é indicada para estimar os diâmetros e representar o afilamento da árvore média da espécie Cordia goeldiana H. A função proposta por Kozak (2004a) mais é adequada em estimar o volume total. Além disso, as equações de forma variável obtiveram ajustes superiores às funções não segmentadas. 

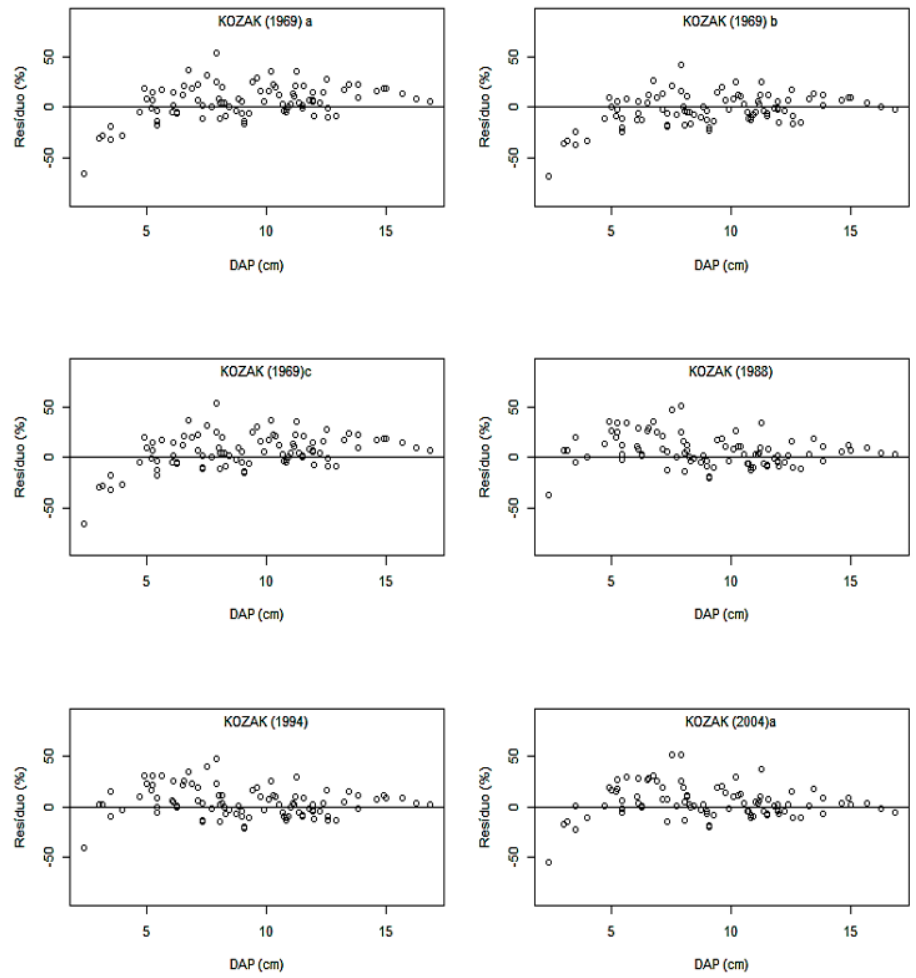

Figura 5. Distribuição dos resíduos (\%) em função de dap (cm) para a estimada de volume total das funções de afilamento não segmentada e de forma variável para o povoamento de Cordia goeldiana $\mathrm{H}$, Vilhena, RO

\section{CONSIDERAÇÕES FINAIS}

O trabalho mostra a forma do fuste da espécie Cordia goeldiana Huber a acuracidade das equações de afilamento de forma variável para esta espécie, e estimula o estudo com outras funções desta categoria utilizando outras espécies

\section{REFERÊNCIAS}

AHRENS, S.; HOLBERT, D. Uma função para forma de tronco e volume de Pinus taeda L. Boletim de Pesquisa Florestal, Curitiba, n. 3,. p.37-68, 1981

ANDRADE, V. C. L. Novos modelos de taper do tipo expoente-forma para descrever o perfil do fuste deárvores. Pesquisa Florestal Brasileira, Colombo, v.34, n.80, p.1-13, 2014. 
ANDRADE, V. C. L.; SCHMITT, T. Modelos de taper empregados em florestas brasileiras nativas e plantadas diferentes do eucalipto e pinus. Advances in Forestry Science, v. 4, n. 1, p. 89-92, 2017.

Assis, A. L. Comparação de modelos polinomiais segmentados e não-segmentados na estimativa de diâmetros e volumes ao longo do fuste de Pinus taeda. Cerne, v. 7, n. 1, p. 20-40, 2001.

ASSIS, A. L. Avaliação de modelos polinomiais não-segmentados na estimativa de diâmetros e volumes comerciais de Pinus taeda. Ciência Florestal, v. 12, n. 1, p. 89-107, 2005.

BARUSSO A. P. A determinação de funções de crescimento mediante análise de tronco. 1977.133f. Dissertação (Mestrado em Ciências Florestais) - Setor de Ciências Agrárias, Universidade Federal do Paraná, Curitiba, 1977.

BASTOS, T. X.; DINIZ, T. D. A. Avaliação do clima do estado de Rondônia para desenvolvimento agrícola. Belém: Embrapa - Centro de Pesquisa do Trópico Úmido, 1982. 27 p. (Boletim de Pesquisa, 44).

CAMPOS, J. C. C.; LEITE, H. G. Mensuração florestal: perguntas e respostas. 3.ed. Viçosa: UFV, 2009. 548 p.

EMBRAPA, Sistema Brasileiro de classificação de solos/ [editores técnicos, Humberto Gonçalves dos Santos -2ª ed. - Rio de Janeiro: Embrapa Solos, 2006. $306 \mathrm{p}$ : il.

FAVALESSA, C. M. C. Funções de afilamento não segmentadas e segmentadas para Tectona grandis na região centro-sul matogrossense. Pesquisa Florestal Brasileira, v. 32, n. 72, p. 373, 2012.

FIGUEIREDO, E. O.; SCOLFORO, J. R. S.; OLIVEIRA, A. D. Seleção de modelos polinomiais para representar o perfil e volume do fuste de Tectona grandis Lf Acta Amazônica VOL. 36(4): 465 - 482. 2006.

FINGER, C. A. G. Fundamentos de biometria florestal. Santa Maria: UFSM, CEPEF - FATEC, 1992, 269 p.

INDUSTRIA BRASILEIRA DE ÁRVORES (IBÁ). Indústria brasileira de árvores. s.l, 2016. 100 p.

KOZAK, A.; MUNRO, D. D.; SMITH, J. H. G. Taper functions and their application in forest inventory. The Forestry Chronicle, v. 45, n. 4, p. 278-283, 1969.

KOZAK, A. A variable-exponent taper equation. Canadian Journal of Forest Research, v. 18, n. 11, p. 1363-1368, 1988.

KOZAK, A. Effects of multicollinearity and autocorrelation on the variable-exponent taper functions. Canadian Journal of Forest Research, v. 27, n. 5, p. 619-629, 1997.

KOZAK, A. My last words on taper equations. The Forestry Chronicle, v. 80, n. 4, p. 507-515, 2004.

MACHADO, S. A. Comparison of methods for estimating heights from completly stem analysis data for Pinus taeda. Ciência Florestal,no prelo.

MACHADO, S. A; FIGUEIREDO, D.J, E. CONCEIÇÃO, M. B. Modelagem de volume individual para diferentes idades e diferentes regimes de desbastes de Pinus 
oocarpa. Revista Ciências Exatas e Naturais. n 2, p. 185-197, 2002

MAX, T. A.; BURKHART, H. E. Segmented polynomial regression applied to taper equations. Forest Science, Washington, v.22, n.3, p.283-289, 1976.

MÔRA, R. Funções de afilamento de forma variável e modelagem de efeitos mistos em fustes de Pinus taeda e Eucalyptus saligna / Rômulo Môra.-Curitiba, 2015. 277f.: il., grafs., tabs.

NEWNHAM, R. M. A variable-form taper function. Can. For. Serv. Petawava Natl. For. Inst. Inf. Rep. PI-X-83., 31 p.,1988.

NEWNHAM, R. M. Variable-form taper functions for four Alberta tree species. Canadian Journal of Forest Research, Ottawa, v.22, n.2, p.210-223, 1992.

PEREZ, D. N.; BURKHART, H. E.; STIFF, C. T. A variable-form taper function for Pinus oocarpa Schiede in Central Honduras. Forest Science, Washington, v.36, n.1, p.186-191, 1990.

QUEIROZ D, MACHADO S. A, FIGUEIREDO-FILHO A., ARCE J. E, KOEHLER H. S. Identidade de modelos em funções de afilamento para Mimosa scabrella Bentham em povoamentos nativos da região metropolitana de Curitiba/PR. Floresta 2008; 38(2): 339-349.

QUEIROZ, D. Avaliação de funções de afilamento e sortimentos para Mimosa scabrella Bentham em povoamentos nativos da região metropolitana de Curitiba/PR. 2006. Tese de Doutorado. Universidade Federal do Paraná.

RAMALHO, A. R.; GODINHO, V. P.; UTUMI, M. M.; JESUS, J. Condicionantes agroclimáticas para a ricinocultura no sul de rondônia. In: CONGRESSO BRASILEIRO DE MAMONA, 1., 2004, Campina Grande. Anais... Campina Grande: Embrapa Algodão, 2004.

RONDÔNIA (ESTADO). Secretaria Estadual de Planejamento. Zoneamento Sócio-Econômico-Ecológico do Estado de Rondônia. Porto Velho: Planafloro, 2000. 21 p. SCOLFORO, J. R. S. THIERSCH, C. R. Biometria florestal: medição e volumetria de árvores. Lavras: UFLA/ FAEPE, 1998. 310p.

SCOLFORO, J. R. S.; THIERSCH, C. R. Biometria Florestal: Medição, Volumetria e Gravimetria. Lavras: UFLA/FAEPE. 2004. 285 p.

SOARES, C. P. B.; PAULA-NETO, F. DE; SOUZA, A. L. DE. Dendrometria e inventário florestal. 2. ed. Viçosa: Ed. UFV, 2011. 272 p.

TÉO, S. J. Funções de afilamento para Pinus taeda L. de diferentes idades, na região de Caçador-SC. In: Anais do 4을 Congresso Florestal Paranaense. 2012.

YOSHITANI-JUNIOR, M., NAKAJIMA, N. Y., ARCE, J. E., MACHADO, S. D. A., DRUSZCZ, J. P., HOSOKAWA, R. T., \& MELLO, A. A. D. Funções de afilamento para plantios desbastados de Pinus taeda. Floresta, Curitiba, PR, v. 42, n. 1, p. 169 - 176, jan./mar. 2012. 